Visual Overview, Oral Detail: The Use of an Emergency-Department Whiteboard

Hertzum, Morten; Simonsen, Jesper

Published in:

International Journal of Human-Computer Studies

DOI:

10.1016/j.ijhcs.2015.04.004

Publication date:

2015

Citation for published version (APA):

Hertzum, M., \& Simonsen, J. (2015). Visual Overview, Oral Detail: The Use of an Emergency-Department Whiteboard. International Journal of Human-Computer Studies, 82, 21-30.

https://doi.org/10.1016/j.ijhcs.2015.04.004 


\title{
Visual Overview, Oral Detail: The Use of an Emergency-Department Whiteboard
}

\author{
Morten Hertzum \\ Royal School of Library and Information Science \\ University of Copenhagen, Denmark \\ hertzum@acm.org
}

\author{
Jesper Simonsen \\ Department of Communication, Business, and Information Technologies \\ Roskilde University, Denmark \\ simonsen@ruc.dk
}

\begin{abstract}
Whiteboards facilitate coordinative practices by making information publicly accessible and thereby strengthening communication and joint commitment about it. This study investigates how coordination is accomplished in an emergency department through interactions with the whiteboard and with the coordinating nurse, who is the main keeper of the whiteboard. On the basis of observations, we find that coordination is accomplished through a highly intertwined process of technologically mediated visual overview combined with orally communicated details. The oral details serve to clarify and elaborate information at a more fine-grained level than the overview information on the whiteboard, to negotiate and reach agreement about the decisions that underlie the whiteboard information, and as a safeguard against misunderstandings and errors. This process is contingent on the clinicians tending to perceive the whiteboard and the coordinating nurse as a unit in the sense that they frequently update the whiteboard by informing the coordinating nurse about the change and, similarly, consider making a change on the whiteboard the same as having informed the coordinating nurse. These smooth transitions between instrumental and communicative coordination are central to the coordinative function of the whiteboard. We discuss this and other implications for design.
\end{abstract}

Keywords: overview, instrumental coordination, communicative coordination, whiteboard, large display groupware, healthcare

\section{Introduction}

Whiteboards serve a coordinative function in much cooperative work by publicly displaying information and facilitating communication about it for purposes such as joint planning and rescheduling (Tang et al., 2009; Whittaker \& Schwarz, 1999; Xiao et al., 2001). Their coordinative function makes whiteboards important artifacts because coordination is central to cooperative work and a frequent cause of breakdowns. While 
shared whiteboards provide access to whiteboard content and opportunity for updating it, it is less clear how the content remains current, attended to, understood, and acted on. To understand this, multiple studies have investigated the work practices associated with whiteboards (e.g., Aronsky et al., 2008; Tang et al., 2009; Whittaker \& Schwarz, 1999). In emergency departments (EDs), whiteboards are integral to the continuous coordination of work (Bjørn \& Hertzum, 2011) and their coordinative function is tightly coupled with the work of the coordinating nurse (Hertzum \& Simonsen, 2013). To avoid errors with potentially adverse effects on patient health, the coordinative practices associated with ED whiteboards must be sufficiently robust to withstand the dynamic, sometimes hectic, ED environment.

In this study we investigate how coordination in an ED is accomplished through interactions with the whiteboard and with the coordinating nurse. We argue, on the basis of workplace observations, that these interactions form a highly intertwined process of technologically mediated visual overview combined with orally communicated details. In short: visual overview, oral detail. Our distinction between interactions with the whiteboard and with the coordinating nurse corresponds to distinctions between instrumental and communicative coordination (e.g., Bardram, 2000). We employ the analytic distinction between instrumental and communicative coordination to show how entangled visual overview and oral detail are in practice. We find, indeed, that the ED clinicians tend to perceive the whiteboard and the coordinating nurse as a unit, rather than as two separate entities. This perception shows how a focus on the whiteboard in isolation is insufficient to understand its coordinative function and leads to a discussion of the coordinative unit consisting of the whiteboard and the coordinating nurse. Our argument can be seen as a cooperative-work note on, or qualification of, Shneiderman's (1996) visual information-seeking mantra ("Overview first, zoom and filter, then detailson-demand"), which presupposes that both overview and details are mediated through instrumental coordination.

In the next section, we describe related work on the coordinative function of whiteboards and elaborate the distinction between instrumental and communicative coordination. Section 3 accounts for the method of our study, which is based on observations at the ED of a Danish hospital. We present our results in Section 4 by showing how the ED clinicians maintain an overview and obtain associated details through interactions with the whiteboard and the coordinating nurse. Section 5 provides a discussion of the intertwined process of visual overview and oral detail, and discusses implications of this process for the design of systems intended to support cooperative work.

\section{Related work}

Shneiderman (1996) presents his visual information-seeking mantra as a guideline for how to design visual interfaces. The mantra, "Overview first, zoom and filter, then details-on-demand", recommends that designers should provide users with an initial overview of the information space and that details should be postponed until users request them. Systems that apply the mantra include Lifelines (Plaisant et al., 1996), which visualizes personal histories as timelines with icons indicating individual events and possibilities for zooming in on specified time periods and for viewing details about specified events. While the data visualized in Lifelines, for example a person's medical history, have been entered by multiple people, the Lifelines system focuses on how to present the data visually to a single user working alone. This focus is consistent with the mantra, which neglects cooperation and is restricted to visual means of conveying 
information. These limitations are not specific to the mantra but appear characteristic of the ways in which the notion of overview is conceptualized in information-visualization research (see, Hornbæk \& Hertzum, 2011).

In contrast, whiteboards exemplify a type of system that supports cooperating actors in their coordinative practices and in maintaining an awareness of the state of their joint work. Studies of such real-time coordination have shown how co-located actors render aspects of their activities visible in order to have others notice and align with actions and events that might otherwise pass unnoticed (e.g., Heath \& Luff, 1992; Hutchins, 1995; Mackay, 1999). When actors are locally mobile within a workplace, such as a hospital, they cannot rely exclusively on oral communication, gazes, and other ephemeral means of coordination. They need artifacts that can, temporarily, hold information and thereby make it visible to others (Whittaker \& Amento, 2003). Whiteboards are widely used for this purpose, partly because they afford making information visually accessible in or near the place where it is needed (Tang et al., 2009).

Whittaker and Schwarz (1999) found that a dry-erase whiteboard located in a public area induced commitment, responsibility, and frequent updates among project participants, who used the whiteboard for collaborative problem solving and project scheduling. The project participants would often gather at the whiteboard to sort things out. Xiao et al. (2001) made similar observations and noted that the sheer size of the studied whiteboard accommodated groups of people standing close to the whiteboard, either discussing or modifying its contents. Cherubini et al. (2007) found that for all the studied purposes of whiteboard use more than half of the survey respondents indicated that multiple people would gather at the whiteboard, thereby emphasizing its collaborative and coordinative function. The purpose with the highest percentage of individual whiteboard use ( 40\%) was 'understanding', suggesting that details were often worked out individually rather than collaboratively. In relation to overview, Hertzum (2011) reported that the replacement of dry-erase with electronic whiteboards at an ED resulted in an improvement in the clinicians' self-reported overview of their work.

Whiteboards are an example of transitional artifacts, which fill a gap between the work being performed and the formal documentation of it in, for example, electronic patient records (Chen, 2010). Electronic patient records support the mandatory documentation of clinical assessments, completed treatments and so forth. Transitional artifacts hold procedural information, present information in accordance with local workflows, or otherwise facilitate the flow of work in ways not done by the formal documentation. Most electronic ED whiteboards resemble dry-erase whiteboards in content and visual layout (Rasmussen, 2012). Both types of whiteboard have a matrix layout with a row for each patient and columns with key information, such as room, patient name, chief complaint, triage level, responsible nurse, and tests ordered. In contrast to the textoriented whiteboards in EDs, electronic whiteboards in surgical suites have been augmented with live video feeds, which show the spatial location of clinicians, indicate how far surgical procedures have progressed, and thereby provide an awareness that facilitates the coordination of upcoming activities (Bardram et al., 2006; Hu et al., 2006). The transitional nature of whiteboards also means that they are more often glanced at in passing than scrutinized in detail (Lederman \& Johnston, 2011) and that they benefit from being centrally located in a department to invite at-a-glance use and information exchange with colleagues (Scupelli et al., 2010).

France et al. (2005) found that an electronic ED whiteboard improved the efficiency of work and communication among the physicians and attributed this improvement to a reduced need for interrupting each other because information previously obtained from 
colleagues had become available on the whiteboard. This study suggested that the introduction of a whiteboard reduced interruptions and oral communication and, thereby, physicians' mental workload. Along similar lines, Aronsky et al. (2008) emphasized that electronic ED whiteboards can provide rapid access to more detailed information by retrieving it from the electronic patient records. Wong et al. (2009) reported that a newly introduced whiteboard brought together information that was previously distributed across multiple people and records. As a result, the clinicians experienced time savings and improved communication. These three studies suggest a reduced need for obtaining information orally because electronic whiteboards make more information readily available.

While the studies mentioned above reported positive effects of whiteboards, other studies have found that the introduction of electronic whiteboards in healthcare settings affected workflows negatively (Pennathur et al., 2007), made clinicians work more individually (Wears et al., 2003), and contained less information relevant to patient treatment (Bisantz et al., 2010). Thus, it is not clear how whiteboard users balance information exchange via the whiteboard against oral information exchange directly with their colleagues. Bardram (2000) conceptualized this balance by distinguishing between instrumental and communicative coordination. Instrumental coordination is mediated by artifacts that temporarily hold information and, for example, includes using a column on a shared whiteboard for holding information about when a patient is ready for transfer to another department, thereby mediating the coordination between the sending department, the receiving department, and the porters physically transferring the patient. Communicative coordination takes place when actors coordinate their activities face to face or by means of communication technologies, which transmit but do not hold information. A nurse may, for example, phone another department and negotiate a time for the transfer of a patient.

Carstensen and Nielsen (2001) compared and contrasted the communicative coordination in maritime navigation with the instrumental coordination in software engineering. In these settings, communicative coordination relied on the use of sentential schemas, which reduced the role of the uttered words to that of providing the important, focal information, while the schemas implicitly supplied the additional information that made the focal information sensible and possible to act on. For example, the command "starboard twenty" would instruct the helmsman to turn the wheel starboard twenty, now. Thus, the schema supplied the information about the subject (the helmsman), the activity (turn), the object (the wheel), and the time (now); only the manner in which the activity should be performed (starboard twenty) was explicitly uttered. Conversely, the artifact in the software engineers' instrumental coordination functioned as a checklist that made it visible what information was required and thereby, to some extent, made the underlying schema explicit. The robustness of the two modes of coordination differed. Communicative coordination afforded smooth shifts between focal information and background schemas, thereby enabling actors to check and, if needed, repair implicit assumptions. Instrumental coordination did not afford such smooth shifts; therefore, uncertainties, mistakes, and other special cases had to be handled by other means. In relation to EDs, a mix of communicative and instrumental coordination is also necessary because ED clinicians, unlike maritime navigators, are locally mobile (Bardram \& Bossen, 2003) and, unlike software engineers, face conditions that change to the extent of making re-coordination a continuous activity (Aronsky et al., 2008).

In summary, previous work shows how artifacts like whiteboards support coordination and overview in situations with single users and situations with multiple collaborating 
actors using the whiteboard. Though these two classes of situation are inclusive, the ED in the present study extends them by adding a situation in which the ED clinicians tend to perceive the whiteboard and the coordinating nurse as a unit. We investigate how this coordinative unit is constituted and how the clinicians interact with this unit, rather than with the whiteboard as such.

\section{Method}

Our study took place at the ED of a medium-size hospital in Region Zealand, one of five healthcare regions in Denmark. The ED comprised 10 patient rooms and consisted of an acute area for patients arriving by ambulance or on referral from their general practitioner and a fast-track area for walk-in patients. The ED saw 40-45 thousand patients a year and was staffed with 25 physicians, 35 nurses, and several secretaries, lab technicians, and department managers. The coordination center of the ED was the control desk, at which the coordinating nurse was stationed. The ED's whiteboard was also located at the control desk (see Figure 1).

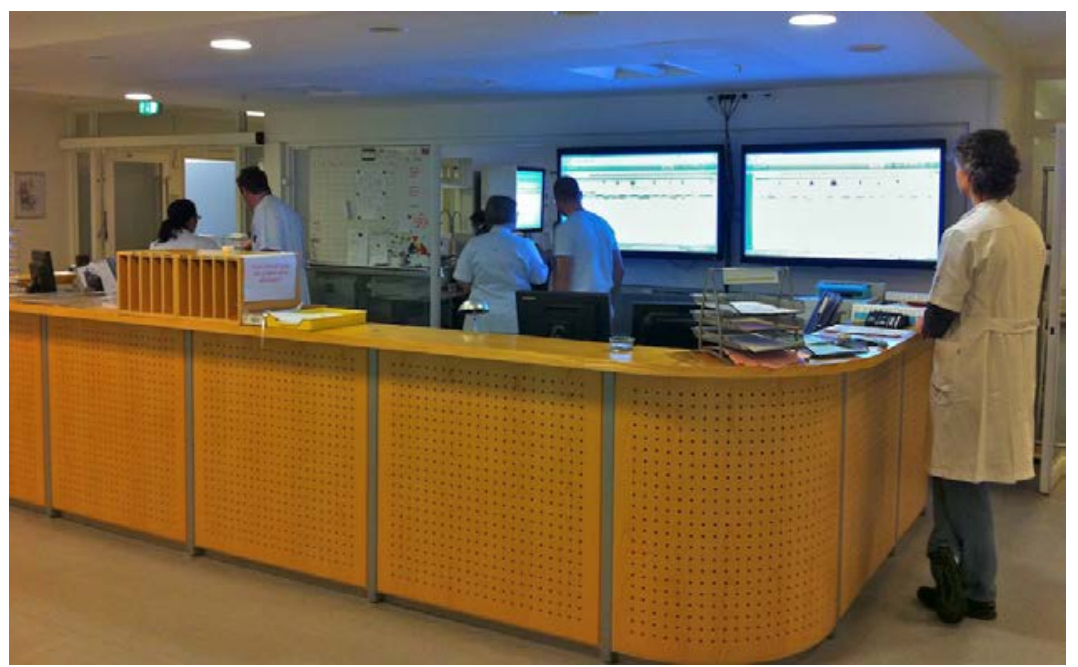

Figure 1. The control desk with the electronic whiteboard (a pair of 52-inch screens)

The data for this study were collected by means of observation supplemented with informal interviews. We chose this means of data collection because coordinative practices and whiteboard use are amenable to observation, because the minutiae in the accomplishment of real ED work were decisive to our study, and because the control desk provided a natural focal point for the observations. The authors observed the work at the ED for a total of 116 hours distributed over two four-week periods. During the first observation period (November 2010) the ED whiteboard was a dry-erase whiteboard; during the second observation period (May 2011) it was an electronic whiteboard, which had been in use for four months. For most of the observations the authors were seated in between some equipment, at a distance of about four meters from the control desk. This position (from which the photo in Figure 1 was taken) provided the authors with a full view of the control desk, including the whiteboard. From this position the authors could also listen in on conversations at the control desk. For the remainder of the observations 
the authors moved around at the ED but did not enter patient rooms when they were in use.

The dry-erase and electronic whiteboards were similar in content and in layout. Both whiteboards gave one row of information about each patient. This information included room, triage level (a five-level color code indicating severity), assigned nurse, assigned physician, status of laboratory tests, next department (or discharge), and porter status. In addition, the electronic whiteboard included information about time of arrival, patient's first name, age, working diagnosis, and current activity. The electronic whiteboard consisted of a pair of wall-mounted, 52-inch screens (see Figure 1). The left-hand screen showed the currently admitted patients and the right-hand screen the patients who had been announced but had not yet arrived. The dry-erase whiteboard was in the location now occupied by the electronic whiteboard and consisted of a pair of back-to-back whiteboards, similarly divided into current and announced patients. Information on the dry-erase whiteboard was handwritten, except the triage level which was indicated by colored magnets. On the electronic whiteboard, information was entered via touch interaction, mostly by selection from dropdown menus. In addition, the electronic whiteboard could be accessed on any computer at the ED and updated using keyboard and mouse. The electronic whiteboard will gradually be integrated with other systems and extended with automatically updated fields of information. Our study was made before such extensions were implemented and at a time where all updates were made manually, thus mirroring the process of updating the dry-erase whiteboard.

We documented our observations in written field notes, which were initially analyzed by reading them thoroughly and comparing different notes. This analysis began while the observations were still going on and, thereby, made it possible to check and elaborate observations and initial interpretations through further observation. The initial analysis led to the identification of a heterogeneous set of issues about the clinicians' interactions with the whiteboard and the coordinating nurse. After the observations had ended these issues were analyzed using the distinction between instrumental and communicative coordination (Bardram, 2000; Carstensen \& Nielsen, 2001), and the theme of visual overview versus oral detail emerged. While the focus on visual overview emerged from observations that the clinicians often merely glanced at the whiteboard, the focus on oral detail emerged from aggregating observations of negotiation, information sharing, consulting, and the like. The remaining analysis was structured around the theme of visual overview versus oral detail and served to consolidate it by identifying additional aspects and examples of it. In the consolidation we paid attention to whether and, if so, how the theme was affected by the shift from dry-erase to electronic whiteboards. Because this shift had little effect on the theme of our analysis we will mostly refer to the whiteboards simply as whiteboards and will only distinguish between dry-erase and electronic whiteboards when the distinction matters to our analysis. We do not mean to discount previous studies documenting subtle differences between dry-erase and electronic whiteboards. A motivation for these studies is that dry-erase and electronic whiteboards are similar in many important respects but different in others. We contend that with respect to the theme of visual overview versus oral detail the dry-erase and electronic whiteboards we studied were similar, for example because they had similar layouts and were updated in similar ways.

Prior to the observations the study was approved by the healthcare region and by the management of the ED. At the beginning of the two observation periods, the authors introduced the observations at the clinicians' morning meetings. Further explanations were provided, on request, during the observations. All names in the field-note excerpts included in this paper are pseudonyms. 


\section{Results}

The coordination of the work in the studied ED revolved around the coordinating nurse and the whiteboard. The coordinating nurse was responsible for maintaining the flow of patients through the ED and for assigning physicians and nurses to patients according to patient needs and clinician workload. These responsibilities meant that the main activities of the coordinating nurse were to receive announcements of the patients about to arrive, to receive the patients arriving by ambulance, to keep an eye on the number of walk-in patients in the waiting room, to find nurses and physicians for the patients, to communicate with other departments at the hospital (e.g., to order laboratory tests and patient transports), and to prioritize and reprioritize the patients on the basis of continuous input from physicians, nurses, and announcements of new patients. One of the coordinating nurse's specific responsibilities was to keep the whiteboard. The role of coordinating nurse circulated among the experienced nurses, who had this role for a full shift or half a shift at a time.

\subsection{The coordinating nurse and the whiteboard}

The coordinating nurse's work was intertwined with the keeping of the whiteboard to the extent that one would be dysfunctional without the other. For this reason alone it was essential that the whiteboard and the coordinating nurse were both located at the control desk in the center of the ED (Figure 1). The following field-note excerpt illustrates the central role of the coordinating nurse in running the ED, the mental and temporal demands inherent in this role, and the tight coupling between the coordinating nurse and the whiteboard:

The coordinating nurse is talking with the coordinating physician while a paramedic arrives with a patient on a stretcher. The paramedic stops at the control desk and awaits instructions. The coordinating nurse finishes her conversation with the coordinating physician (about 10 seconds) and then turns to the paramedic: 'This is Mary?' The paramedic confirms and adds: 'She has been kicked by a horse'. The coordinating nurse instructs the paramedic to take the patient to Room 9, which is currently unoccupied on the whiteboard.

As the paramedic wheels the patient into Room 9, the coordinating nurse has the outstanding tasks of recording the patient on the whiteboard and finding a nurse who has time to see the patient. However, a lab technician just arrives and asks the coordinating nurse whether the patient in Room 2 is ready for his blood test. The coordinating nurse confirms. Also, two porters pass the control desk with a patient in a bed and announce: 'We are leaving with Room 5'. The coordinating nurse now also needs to record on the whiteboard that the patient in Room 5 has left for EKG. She is however interrupted by the phone, picks it up, and talks for 20-30 seconds. As the phone call is coming to an end, the coordinating nurse moves over to the whiteboard and records that there is now a patient in Room 9, but no information is recorded about the priority of the patient, nor has a nurse yet been assigned to the patient.

The coordinating nurse makes a call on the phone. While she waits for the callee to pick up she first scans the whiteboard then the area around the control desk to locate a free nurse. Then the phone is picked up and she talks for 10-20 seconds. As she ends the call the nurse responsible for Room 5 passes the control desk and the coordinating nurse informs her that the patient has been taken to EKG. She simultaneously records this information on the whiteboard. Afterward, the coordinating nurse leaves the control desk to take a look down the hallway and then returns to the control desk [presumably she looked for a free nurse, in vain]. A little later a nurse passes and the coordinating nurse asks her whether she can take Room 9. The nurse confirms, and the coordinating nurse records this on the whiteboard. The seriousness of the injury of the patient in Room 9 has yet to be determined, as indicated by the absence of information about the priority of this patient. [Nov 3, 2010 - dry-erase whiteboard] 
The coordinating nurse partly recorded information on the whiteboard to maintain her own overview of the state of the ED and partly to make it readily available to the other clinicians. While the whiteboard should, in principle, always be up to date, the excerpt shows that updates were frequently delayed because the coordinating nurse was interrupted.

\subsection{Instrumental coordination}

The whiteboard provided the clinicians with a general overview of the state of the ED and with specific information about their own current tasks. Most interactions with the whiteboard were brief. The location of the whiteboard at the control desk supported such interactions because the clinicians visited the control desk many times during a shift, for example to pick up papers about their current patient, to consult more experienced colleagues, to make themselves available for consultation, and simply in passing back and forth between the patient rooms, the physician work area, the waiting room, and the staff room. When they approached the control desk the clinicians typically glanced at the whiteboard. Often they did not appear to be looking for anything in particular but rather to be ascertaining whether everything was in hand or something was out of the ordinary. The following excerpt illustrates one such interaction with the whiteboard and also that these interactions mostly led to the conclusion that everything was in hand and thereby that the clinician could confidently continue his or her current activity:

A physician at the control desk phones someone at another department. While he waits for the callee to pick up the phone, he scans the whiteboard. When the callee answers, the physician leaves the control desk and walks down the hallway. [May 11, 2011 - electronic whiteboard]

On other occasions the clinicians' interactions with the whiteboard were similarly brief but they were looking for specific pieces of information. These interactions often concerned determining the room number of a patient, checking the status of previously ordered laboratory tests, or - as in the following excerpt - identifying the nurse or physician responsible for a patient:

A nurse passes through the control desk and as she passes the whiteboard she locates the name of the physician responsible for the patient in Room 2. As soon as she enters the physician work area, which adjoins the control desk, she says 'Cecilie' and starts talking directly to the physician responsible for the patient in Room 2, among the three physicians in the physician work area. [Nov 11, 2010 - dry-erase whiteboard]

In addition to using the information on the whiteboard for maintaining their overview of the state of the ED, the clinicians also interacted with the whiteboard to update its content. Though the coordinating nurse made the majority of the updates, the transition from dry-erase to electronic whiteboards was accompanied by an increase in the number of updates made by other clinicians. During our informal interviews, a clinician expressed that some coordinating nurses welcomed updates made by other clinicians whereas others preferred to make the updates themselves. Importantly, the clinician stated that this difference had not surfaced with the introduction of the electronic whiteboard but had also been present during the use of the dry-erase whiteboard. The increase in the number of updates made by other clinicians after the transition to the electronic whiteboard was partly brought about by the possibility for distributed access to the whiteboard. With the electronic whiteboard the secretaries, for example, started to enter initial whiteboard information about the walk-in patients from their computers in the secretaries' office. After the introduction of the electronic whiteboard, updates were, to some extent, also made by physicians, nurses, lab technicians, and occasionally porters, even though they needed to log on before they could make changes. To simplify 
the logon process all clinicians carried a personal token and logged on by briefly holding this token onto a sensor next to the whiteboard. A simple and swift logon process was essential because the clinicians typically logged on to make a single update and then left the whiteboard again:

A lab technician has just drawn blood from the patient in Room 3. On leaving with the blood sample, she passes the control desk, logs on to the whiteboard, and changes the status of the blood test from 'ordered' to 'taken'. [May 11, 2011 - electronic whiteboard]

Instrumental coordination in terms of interactions with the whiteboard was often rendered near invisible by the brevity of the interactions, especially when the clinicians merely read information off the whiteboard by glancing at it in passing. This invisibility might lead to underestimation of the amount and effectiveness of the instrumental coordination, which was most visible when it broke down:

Physician A examines the EKG for the patient in Room 1 and then glances at the whiteboard, on which no physician is recorded as responsible for Room 1. Physician A therefore goes to Room 1 where the patient and his relatives inform her that the patient has already been seen by a physician. Physician A responds that it probably was a nurse, but the patient maintains that he has been seen by a physician, 'a thin, blond physician'. Physician A returns to the control desk and rechecks the whiteboard, then she asks out loud whether there has already been a physician in Room 1. The coordinating nurse is temporarily away from the control desk, and the clinicians present do not respond. As Physician A is leaving the control desk, Physician B passes by and Physician A asks her whether she has Room 1. Physician B, who is thin and blond, confirms. Five minutes later it has still not been recorded on the whiteboard that Physician B is responsible for the patient in Room 1. [May 19, 2011 - electronic whiteboard]

In the excerpt above instrumental coordination broke down because the whiteboard information was not current. In other situations the clinicians turned from instrumental to communicative coordination because they needed richer information than the terse pieces of information on the whiteboard.

Table 1. Number of changes to the dry-erase and electronic whiteboards

\begin{tabular}{lcccccc}
\hline \multirow{2}{*}{ Time of day } & \multicolumn{2}{c}{ Dry-erase whiteboard } & & \multicolumn{2}{c}{ Electronic whiteboard } \\
\cline { 2 - 3 } \cline { 5 - 6 } & Hours of data & Hourly changes & & Hours of data & Hourly changes \\
\hline 00:00-09:59 & 2 & & & & $10 \times 28$ & 40.9 \\
10:00-10:59 & 3 & 27.3 & & 28 & 109.0 \\
11:00-11:59 & 4 & 31.3 & & 28 & 103.3 \\
12:00-12:59 & 4 & 41.8 & & 28 & 103.3 \\
13:00-13:59 & 4 & 50.0 & & 28 & 111.0 \\
14:00-14:59 & 4 & 35.6 & & 28 & 101.7 \\
15:00-15:59 & 5 & 38.0 & & 28 & 85.4 \\
16:00-16:59 & 1 & 38.0 & & 28 & 81.8 \\
17:00-17:59 & 1 & & & $6 \times 28$ & 80.9 \\
18:00-23:59 & & 35.2 & & 58.4 \\
\hline Hourly average & &
\end{tabular}

Note. To compress the table we have collapsed the data for the first ten hours of the day into one row and the data for the last six hours of the day into one row. 
To give a sense of the amount of instrumental coordination we extracted data about the number of updates from the log of the electronic whiteboard over the four-week observation period. We supplement these data with 24 hours of observations of the dryerase whiteboard during which we manually registered every time the whiteboard content was changed. Table 1 shows the results. The electronic whiteboard was updated about once a minute around the clock. During the daytime hours for which we have data for the dry-erase whiteboard, it was updated about once every two minutes. A comparison of the update frequencies is merely suggestive because the larger number of fields of information on the electronic whiteboard accounted for some of the difference.

\subsection{Communicative coordination}

As illustrated in the previous excerpt the clinicians resorted to communicative coordination when instrumental coordination broke down. The possibility of sorting out omissions on the whiteboard through communicative coordination was an important safeguard against misunderstandings and errors, and it shows the robustness of communicative coordination in the face of breakdowns. However, in most situations communicative coordination was not triggered by breakdowns.

The clinicians perceived the coordinating nurse and the whiteboard as a unit in the sense that they frequently updated the whiteboard by informing the coordinating nurse about the change rather than by changing the whiteboard content themselves. Similarly, they considered making a change on the whiteboard the same as having informed the coordinating nurse. The clinicians mostly informed the coordinating nurse about changes and left it to him or her to update the whiteboard. This way the clinicians had an efficient and hands-free 'interface' to the whiteboard. While this interface fitted well with many of the clinicians' tasks, it increased the load on the coordinating nurse's memory and engaged the coordinating nurse in near constant multitasking:

A porter arrives to pick up the patient in Room 2. When the porter wheels the bed with the patient passed the control desk on his way out he just says: 'We are leaving'. The coordinating nurse is engaged in a phone conversation at the other end of the control desk, but five minutes later she has updated the whiteboard, which now reflects that the patient in Room 2 has been picked up. [Nov 3, 2010 - dry-erase whiteboard]

The changes to be communicated to the coordinating nurse were often not sufficiently important to warrant a visit to the control desk for the sole purpose of informing the coordinating nurse about the change. Rather, the clinicians informed the coordinating nurse when they passed the control desk for other purposes. This meant that they were mentally engaged in other activities and often also carried items in their hands. On a couple of occasions we observed physicians who updated the whiteboard while holding a phone in one hand and some papers in the other. However, on most occasions before as well as after the transition to the electronic whiteboard, physicians and other clinicians preferred the simplest way of handing over information and therefore orally informed the coordinating nurse:

In passing the control desk on his way to Room 10 a nurse says: 'When he arrives then give him Room 7'. It is entirely implicit whom the nurse is referring to but the coordinating nurse replies with a 'yes' and records the room assignment on the whiteboard. [May 11, 2011 electronic whiteboard]

To be able to keep the whiteboard the coordinating nurse needed more than information from the clinicians; the coordinating nurse also needed their agreement and commitment because the whiteboard content reflected numerous interrelated decisions about how to prioritize patients, clinicians, and other resources. Thus, decisions about, for example, 
which physician and nurse were to become responsible for a patient had to be negotiated and, at times, renegotiated. Such negotiations were made through communicative coordination, which in our observations always accompanied the recording of a decision on the whiteboard:

The coordinating nurse and another nurse (Nurse A) stand at the control desk talking about the patient in Room 10, for whom Nurse A is currently responsible.

Nurse B (also at the control desk): Just ask me about Room 10. She is [describes the patient].

Nurse A: Can you take her from here? I got her from [another nurse] but my shift ends at 3 [the time is now 5 minutes past 3]. You can talk to [a specified physician] about her. He was on the phone about her, but then he left.

Nurse B: What's going to happen with her?

Nurse A: She has been [mentions the treatment that has taken place]

Nurse B accepts to assume responsibility for the patient in Room 10, and the coordinating nurse is aware of this because she has been overhearing the conversation. [Nov 2, 2010 - dryerase whiteboard]

On other occasions instrumental coordination, such as overseeing an update of the whiteboard, triggered communicative coordination, which informed the coordinating nurse about events and circumstances that were important to the coordination of the work in the ED but not represented as information on the whiteboard. This way, communicative coordination was frequently used for information below the level of detail recorded on the whiteboard:

The coordinating nurse is deleting the patient in Room 5 from the whiteboard because a porter has just left with the patient. A nurse standing at the control desk observes this and says: 'I'll go and clean Room 5'. The temporary unavailability of the room as well as the distinction between an empty and an empty-and-ready (i.e., clean) room is below the level of detail recorded on the whiteboard; it is remembered by the coordinating nurse. [May 11, 2011 electronic whiteboard]

Finally, the whiteboard was central to the handover from one coordinating nurse to the next. The handover differed from other interactions with the whiteboard in duration and in the intensity of its focus on the whiteboard. Ensuring that the whiteboard was current was an important element of the handover because discrepancies between the whiteboard and the state of the ED would be lost when the current coordinating nurse, who would mostly be aware of such discrepancies, left. The handover was also an example of tightly coupled instrumental and communicative coordination in that whiteboard content triggered communication just as communication triggered whiteboard updates:

The current coordinating nurse and the on-coming coordinating nurse are spending five minutes walking through the patients on the whiteboard. Initially the current coordinating nurse is updating the whiteboard as they speak, but gradually the on-coming coordinating nurse performs more and more of the updates. At the end of the five minutes the on-coming coordinating nurse has assumed responsibility for the whiteboard. [Nov 23, 2010 - dry-erase whiteboard]

\section{Discussion}

The whiteboard and the coordinating nurse are tightly coupled and collectively coordinate the work in the ED. Our contribution is to analyze how this coordinative unit comprises an effective and efficient means of coordinating ED work by intertwining visual overview and oral detail. We consider this analysis significant because the coordinative unit of whiteboard and coordinating nurse is pivotal to the work in the ED 
and, more broadly, exemplifies the intricacy with which communicative and instrumental means are blended in the accomplishment of cooperative work. In the following we discuss visual overview, oral detail, the coordinative unit consisting of the whiteboard and the coordinating nurse, and the implications of this study for design.

\subsection{Visual overview}

The whiteboard provides an immediate visual overview of the state of the ED. By glancing briefly at the whiteboard the clinicians know whether everything is in hand. If so, they can continue with their current activities; otherwise they turn to communicative coordination. The frequent whiteboard updates (Table 1) along with a generally positive attitude toward the whiteboard (Hertzum, 2011; Hertzum \& Simonsen, 2013) indicate that this visual overview is effective.

The visual overview is in part conveyed by the whiteboard layout, which stipulates what information the whiteboard contains and where this information is located on the whiteboard. As pointed out by Carstensen and Nielsen (2001) these stipulations denote that empty whiteboard fields are not devoid of information but indicate work yet to be done and decisions yet to be made. Thus, the pattern of filled-in and empty fields for a patient gives an immediate impression of how far the patient has progressed along his or her trajectory in the ED. Clinicians are proficient at reading such patterns. For example, Nygren et al. (1998) showed that even when the content of a well-structured laboratory form was purposefully made illegible the form still conveyed considerable information to physicians who could see which parts of the form contained information and knew the type of information that went in the different parts. In this respect ED whiteboards with their matrix layout differ from surgical whiteboards with live video feeds (e.g., Hu et al., 2006) because the video feed adds no structure to the streamed activities. For example, the absence of activity on the live video feed from an operating theater does not in itself indicate whether an operation is delayed or no operation is scheduled.

With specific reference to the studied ED whiteboard, its layout (common to the dryerase and electronic whiteboards) means that the number of whiteboard rows that contain information corresponds to the number of patients and thereby gives a visual overview of the occupancy level of the ED. This overview is further improved by dividing the whiteboard into separate displays of the currently admitted patients (on the left) and the announced patients (on the right). Thus, a quick look at the whiteboard tells the clinicians how busy their colleagues currently are and how their collective level of busyness will develop in the near future. When the occupancy level is high, or about to get high, the pattern of filled-in and empty fields for the admitted patients shows how strained the ED is. If all patients have a nurse and a physician assigned then the ED is coping with the busyness and the clinicians can stay focused on the patients to which they are assigned. If, on the other hand, multiple patients do not have a physician or nurse assigned then a clinician glancing at the whiteboard will know that the coordinating nurse is struggling to maintain the flow of patients through the ED. In such situations the atmosphere becomes tense due to the pressure caused by the busyness and due to the associated risk of being unable to accommodate new patients with an acute need for treatment.

The whiteboard also provides a visual overview by rendering selected information persistently available. This double function of remembering and displaying the information is crucial in a work environment where the clinicians are locally mobile, yet need to coordinate their activities. The whiteboard tends, however, to provide pointers to the needed information, rather than to supply the information itself. As analyzed by 
Bjørn and Hertzum (2011) ED whiteboards, for example, supply information about the status of ordered laboratory tests. This information helps the physicians coordinate their work with the work of the lab by aligning their examination of a patient with the arrival of test results. Test results are, however, not on the whiteboard but must be looked up in another system. This way, the whiteboard specifically supports the coordination of ED work; it does not itself supply the information needed to perform ED work. Similarly, the whiteboard facilitates access to the physician and nurse assigned to a patient by displaying their names; this information points to people who can provide more detail on the patient's condition and treatment.

\subsection{Oral detail}

The communicative coordination between the coordinating nurse and the other clinicians mainly elaborates information at a level of detail not included on the whiteboard but necessary to be able to act on the whiteboard information. This way, the communicative coordination shapes the clinicians' understanding of the whiteboard information, which, in turn, shapes the communicative coordination. Such co-varying of recordings and actions - in this case whiteboard information and communicative coordination - has previously been discussed by Wærn (1998), who notes that in dynamic contexts recordings may quickly become outdated and, as a result, misinform action. The communicative coordination serves to negotiate and reach agreement about the decisions that underlie the whiteboard information. When we asked clinicians during our observations they indicated that communicative coordination generally precedes all resource allocations recorded on the whiteboard. Communicative coordination is flexible and enables the clinicians to communicate about any detail relevant to the coordination of their work. It provides rapid feedback and facilitates collaborative exploration of relevant issues by quickly making many communicative turns.

An important function of the communicative coordination is to enable the clinicians to pass on changes to whiteboard information without interrupting their current activities. Typically this happens when the clinicians, in passing the control desk, orally inform the coordinating nurse about the change. Like in Carstensen and Nielsen's (2001) study of maritime navigation, the ED clinicians' shared local context means that much remains implicit in the communicative coordination. For example, the porter's announcement that 'We are leaving' makes sense as an instance of a specific communicative schema, and it is by recognizing this schema the coordinating nurse becomes able to align the explicit information in the porter's announcement with the implicit information from their shared context. While this way of orally communicating details is generally robust it is, for central pieces of information, further consolidated by the duplication of this information on the whiteboard. This duplication resembles the echoing of received commands in maritime navigation and provides a way for the clinicians to verify that information delivered to or received from the coordinating nurse has been understood correctly. The use of the whiteboard for echoing makes it possible to perform the communicative coordination and the verification asynchronously, which is beneficial in an environment where the clinicians are locally mobile and often communicate with the coordinating nurse when they happen to pass the control desk as part of other activities.

Communicative coordination also sorts out omissions and errors on the whiteboard and may thereby resolve breakdowns. The face-to-face nature of the communicative coordination means that the oral details are supplemented by rich back-channeling, for example during the handovers from one coordinating nurse to the next. This backchanneling (Krauss \& Fussell, 1990) increases redundancy and decreases the risk that 
misunderstandings go unnoticed. A further reduction of this risk is the high frequency with which other clinicians are present at the control desk and overhear exchanges of oral details between the coordinating nurse and another clinician. The control desk accommodates the presence of multiple clinicians by being a centrally located, spacious area (see also, Scupelli et al., 2010). In addition, the desk itself provides something to lean against as well as support for papers and other artifacts, thereby affording the oral exchange of details.

\subsection{A coordinative unit}

Visual overview and oral detail form an intertwined process that is facilitated by smooth transitions between instrumental and communicative coordination. With smooth transitions, clinicians can effortlessly and frequently shift between visual overview and oral detail. The co-location of the whiteboard and the coordinating nurse at the control desk is essential in this respect. In the studied ED, the smooth transitions blurred the distinction between the coordinating nurse and the whiteboard to the extent that the clinicians tended to perceive them as a unit. Communicative coordination frequently served as a proxy for instrumental coordination, and vice versa, in that the whiteboard was updated by informing the coordinating nurse or, conversely, the coordinating nurse was informed by updating the whiteboard. We contend that the clinicians predominantly perceived a dyadic relationship between themselves and the coordinative unit, rather than a triadic relationship between themselves, the coordinating nurse, and the whiteboard. The coordinative unit simply had two interfaces, a communicative and an instrumental.

In a study of patients' relationship with their general practitioner (GP) during consultations, Pearce et al. (2011) distinguished between dyadic relationships, in which patients related to the GP and largely ignored the GP's computer, and triadic relationships, in which patients related to both GP and computer. While EDs are obviously different from general practice, our finding of a dyadic relationship contests that the situation necessarily consists of three parties. In the study by Pearce et al., the situations with a dyadic relationship still had three parties, but one of them was consistently ignored by the patient. The triadic relationships found by Pearce et al. often involved that the patients invoked the GP's computer to break impasses, thereby treating the computer as a source of information independent of - and possibly superior to - the GP. When the coordinating nurse and the whiteboard were perceived as independent parties in our study, it was mainly in situations of breakdown, for example when out-ofdate whiteboard information caused confusion among clinicians. There were also examples, with the dry-erase whiteboard as well as the electronic whiteboard, of dyadic relationships in the sense of Pearce et al. but these examples mostly involved people, such as paramedics, who visited the ED briefly and intermittently. The ED staff tended to perceive a dyadic relationship between themselves and a coordinative unit.

Maintaining the unit of whiteboard and coordinating nurse is a demanding task in the fast-paced ED setting, and it largely prevents the coordinating nurse from seeing patients. In less dynamic, less health-critical settings, smooth transitions between instrumental and communicative coordination will be more of a collective responsibility because it will not be considered resource efficient to station a person by the whiteboard. In such settings, changes to the whiteboard content must instead be aligned with the presence of other people at the whiteboard or other people must subsequently be located to inform them about the presence of changes in the whiteboard content. Such aligning and locating is a recurrent activity in many work settings (Bardram \& Bossen, 2003) and 
makes the coordinative function of a whiteboard more effortful by dissociating the oral details from the visual overview.

\subsection{Implications for design}

We see five implications of our analysis for the design of systems that, like whiteboards, are intended to support actors in coordinating their cooperative work.

First, in collaborative settings different modes of coordination may be preferred for overview and detail information. Whiteboards match this differential preference by restricting the overview information to a few carefully selected pieces of information. In sustaining this restriction, it is probably a benefit that ED whiteboards are transitional artifacts (Chen, 2010) because this implies a separation between the whiteboard and the electronic patient record, which contains all the details required in the formal documentation. Increased possibilities for populating whiteboards with information automatically received from other systems will improve the possibilities for keeping the whiteboard information current but will also make it denser. The risk to be considered is that the whiteboard may start to require scrutiny and, thus, no longer provide the clinicians with an at-a-glance overview.

Second, the unit of the whiteboard and the coordinating nurse is important to the coordination of work in the ED but may become strained by automatic whiteboard changes, which risk leaving the coordinating nurse out of the loop. To counteract this risk the way in which automatic whiteboard changes are visualized must be carefully designed. One possibility is that when the coordinating nurse logs on to the whiteboard then changes since her or his last logon are highlighted. Another possibility, utilizing that the coordinating nurse is stationed at the whiteboard, could be to animate automatic whiteboard changes, briefly and discretely. The visualizations must be lightweight to support overview rather than create new work, such as acknowledgements of having seen changes.

Third, a visual overview on a shared whiteboard broadcasts the overview information as well as makes the underlying schema at least partially visible. The schema is an important part of the conveyed information because it adds structure and thereby makes the location of information on the whiteboard meaningful (Nygren et al., 1998). This way, the pattern of filled-in and empty whiteboard fields conveys information important to the visual overview, even without reading the content of the filled-in fields. For location to remain meaningful the schema must remain stable over time and across platforms, such as when overview information is accessed on a handheld device rather than the whiteboard.

Fourth, the ways in which systems provide access to detail must take into account where the needed detail can be obtained. Shneiderman (1996) presupposes that detail is electronically available on demand, but many details exist only as a negotiated mutual understanding among the people involved in an activity. Access to such details requires communicative coordination, thereby making the conditions for communicative coordination part of systems design. Large whiteboard displays contribute to these conditions by creating a place for people to meet, or arrange to meet, but the physical surroundings of the whiteboard must accommodate such meetings.

Finally, it is important to design for smooth transitions between visual overview and oral detail. This means considering, as part of the design, how to make communicative coordination happen in the vicinity of the whiteboard. The combination of instrumental and communicative coordination serves not just to communicate a wider spectrum of 
information but also to bolster its quality, for example by detecting whiteboard errors or misinterpreted patient symptoms through information exchanges with other clinicians.

\subsection{Limitations}

Three limitations should be remembered in interpreting the results of this study. First, all updates of the electronic whiteboard were made manually. We acknowledge that manual updating is a transitional state, which in the future will be supplemented by automatically receiving information from other systems. The introduction of automatic updates will necessitate renewed analysis. In our case, the dry-erase and electronic whiteboards were similar with respect to the theme of visual overview versus oral detail and hence we conflated the data about the two types of whiteboard into one analysis. Second, we have only 24 hours of data about the number of changes on the dry-erase whiteboard (Table 1), all collected on weekdays. These data are suggestive only and merely intended to provide a context for the four weeks of data about the number of changes on the electronic whiteboard. Third, we do not have quantitative data about who updated the whiteboards. We acknowledge that such data would strengthen our argument that the coordinating nurse made most whiteboard changes also on the electronic whiteboard.

\section{Conclusion}

Our observations at the ED reveal a coordinative practice that blends instrumental and communicative coordination. Rather than obtaining details-on-demand through instrumental coordination, the coordinative practice consists of visual overview intertwined with oral details. Visual overview is mediated by the whiteboard, which temporarily holds information, provides for at-a-glance reading, and echoes information communicated to the coordinating nurse. Oral detail is obtained through communicative coordination with the coordinating nurse and serves to clarify information, reach agreement, and safeguard against error. The ED clinicians tend to perceive the whiteboard and the coordinating nurse as a coordinative unit, which can be accessed communicatively as well as instrumentally. Smooth transitions between communicative and instrumental coordination are essential to the intertwined process of visual overview and oral detail. In a collaborative setting such as the ED, much information exists only as a shared understanding among the clinicians. Attempting to rely on the whiteboard for details-on-demand would imply a grave underappreciation of this shared understanding and of the qualities of communicative coordination. In a work setting characterized by local mobility, the whiteboard contributes to this communicative coordination by creating a place for clinicians to meet.

\section{Acknowledgements}

This study is part of the Clinical Overview project, which was conducted in collaboration with the healthcare region Region Zealand and the whiteboard vendor Imatis and co-funded by Vækstforum Sjælland and Innovasjon Norge. The authors are neither financially, personally, nor otherwise related with Region Zealand and Imatis, apart from the professional relations that have evolved in the course of the project. The funding sources have had no role in designing, carrying out, and writing about the study. Special thanks are due to the ED clinicians, who in spite of their busy schedule were accommodating toward our presence. 


\section{References}

Aronsky, D., Jones, I., Lanaghan, K., \& Slovis, C. M. (2008). Supporting patient care in the emergency department with a computerized whiteboard system. Journal of the American Medical Informatics Association, 15(2), 184-194.

Bardram, J. (2000). Temporal coordination - On time and coordination of collaborative activities at a surgical department. Computer Supported Cooperative Work, 9(2), 157-187.

Bardram, J., \& Bossen, C. (2003). Moving to get ahead: Local mobility and collaborative work. In ECSCW 2003: Proceedings of the European Conference on Computer Supported Cooperative Work (pp. 355-374). Amsterdam: Kluver.

Bardram, J., Hansen, T., \& Soegaard, M. (2006). AwareMedia: A shared interactive display supporting social, temporal, and spatial awareness in surgery. In Proceedings of the CSCW'06 Conference on Computer Supported Cooperative Work (pp. 109-118). New York: ACM Press.

Bisantz, A. M., Pennathur, P. R., Guarrera, T. K., Fairbanks, R. J., Perry, S. J., Zwemer, F., \& Wears, R. L. (2010). Emergency department status boards: A case study in information systems transition. Journal of Cognitive Engineering and Decision Making, 4(1), 39-68.

Bjørn, P., \& Hertzum, M. (2011). Artefactual multiplicity: A study of emergency-department whiteboards. Computer Supported Cooperative Work, 20(1\&2), 93-121.

Carstensen, P. H., \& Nielsen, M. (2001). Characterizing modes of interaction: A comparison between oral and artifact based coordination. In Proceedings of the GROUP01 Conference on Supporting Group Work (pp. 81-90). New York: ACM Press.

Chen, Y. (2010). Documenting transitional information in EMR. In Proceedings of the CHI2010 Conference on Human Factors in Computing Systems (pp. 1787-1796). New York: ACM Press.

Cherubini, M., Venolia, G., DeLine, R., \& Ko, A. J. (2007). Let's go to the whiteboard: How and why software developers use drawings. In Proceedings of the CHI 2007 Conference on Human Factors in Computing Systems (pp. 557-566). New York: ACM Press.

France, D. J., Levin, S., Hemphill, R., Chen, K., Rickard, D., Makowski, R., Jones, I., \& Aronsky, D. (2005). Emergency physicians' behaviors and workload in the presence of an electronic whiteboard. International Journal of Medical Informatics, 74(10), 827-837.

Heath, C., \& Luff, P. (1992). Collaboration and control: Crisis management and multimedia technology in London Underground line control rooms. Computer Supported Cooperative Work, 1(1\&2), 69-94.

Hertzum, M. (2011). Electronic emergency-department whiteboards: A study of clinicians' expectations and experiences. International Journal of Medical Informatics, 80(9), 618-630.

Hertzum, M., \& Simonsen, J. (2013). Work-practice changes associated with an electronic emergency department whiteboard. Health Informatics Journal, 19(1), 46-60.

Hornbæk, K., \& Hertzum, M. (2011). The notion of overview in information visualization. International Journal of Human-Computer Studies, 69(7\&8), 509-525.

Hu, P. F., Xiao, Y., Ho, D., Mackenzie, C. F., Hu, H., Voigt, R., \& Martz, D. (2006). Advanced visualization platform for surgical operating room coordination: Distributed video board system. Surgical Innovation, 13(2), 129-133.

Hutchins, E. (1995). Cognition in the wild. Cambridge, MA: MIT Press.

Krauss, R. M., \& Fussell, S. R. (1990). Mutual knowledge and coomunicative effectiveness. In J. Galegher, R. E. Kraut \& C. Egido (Eds.), Intellectual Teamwork: Social and Technological Foundations of Cooperative Work (pp. 111-145). Hillsdale, NJ: Erlbaum.

Lederman, R., \& Johnston, R. B. (2011). Decision support or support for situated choice: Lessons for system design from effective manual systems. European Journal of Information Systems, 20(5), 510-528.

Mackay, W. E. (1999). Is paper safer? The role of paper flight strips in air traffic control. ACM Transactions on Computer-Human Interaction, 6(4), 311-340.

Nygren, E., Wyatt, J. C., \& Wright, P. (1998). Helping clinicians to find data and avoid delays. Lancet, 352(9138), 1462-1466. 
Pearce, C., Arnold, M., Philips, C., Trumble, S., \& Dwan, K. (2011). The patient and the computer in the primary care consultation. Journal of the American Medical Informatics Association, 18(2), 138-142.

Pennathur, P. R., Bisantz, A. M., Fairbanks, R. J., Perry, S. J., Zwemer, F., \& Wears, R. L. (2007). Assessing the impact of computerization on work practice: Information technology in emergency departments. In Proceedings of the Human Factors and Ergonomics Society 51st Annual Meeting (pp. 377-381). Santa Monica, CA: HFES.

Plaisant, C., Milash, B., Rose, A., Widoff, S., \& Shneiderman, B. (1996). LifeLines: Visualizing personal histories. In Proceedings of the CHI'96 Conference on Human Factors in Computing Systems (pp. 221-227). New York: ACM Press.

Rasmussen, R. (2012). Electronic whiteboards in emergency medicine: A systematic review. In Proceedings of the IHI2012 International Health Informatics Symposium (pp. 483-492). New York: ACM Press.

Scupelli, P., Xiao, Y., Fussell, S. R., Kiesler, S., \& Gross, M. D. (2010). Supporting coordination in surgical suites: Physical aspects of common information spaces. In Proceedings of the CHI 2010 Conference on Human Factors in Computing Systems (pp. 1777-1786). New York: ACM Press.

Shneiderman, B. (1996). The eyes have it: A task by data type taxonomy for information visualizations. In Proceedings of the 1996 IEEE Conference on Visual Languages (pp. 336343). Los Alamitos, CA: IEEE Press.

Tang, A., Lanir, J., Greenberg, S., \& Fels, S. (2009). Supporting transitions in work: Informing large display application design by understanding whiteboard use. In Proceedings of the GROUP2009 Conference on Supporting Group Work (pp. 149-158). New York: ACM Press.

Wears, R. L., Perry, S. J., Shapiro, M., Beach, C., Croskerry, P., \& Behara, R. (2003). A comparison of manual and electronic status boards in the emergency department: What's gained and what's lost? In Proceedings of the Human Factors and Ergonomics Society 47th Annual Meeting (pp. 1415-1419). Santa Monica, CA: HFES.

Whittaker, S., \& Amento, B. (2003). Seeing what you are hearing: Co-ordinating responses to trouble reports in network trobleshooting. In K. Kuutti, E. H. Karsten, G. Fitzpatrick, P. Dourish \& K. Schmidt (Eds.), ECSCW2003: Proceedings of the Eighth European Conference on Computer Supported Cooperative Work (pp. 219-238). Heidelberg: Springer.

Whittaker, S., \& Schwarz, H. (1999). Meetings of the board: The impact of scheduling medium on long term group coordination in software development. Computer Supported Cooperative Work, 8(3), 175-205.

Wong, H. J., Caesar, M., Bandali, S., Agnew, J., \& Abrams, H. (2009). Electronic inpatient whiteboards: Improving multidisciplinary communication and coordination of care. International Journal of Medical Informatics, 78(4), 239-247.

Wærn, Y. (1998). Analysis of a generic dynamic situation. In Y. Wærn (Ed.), Co-Operative Process Management: Cognition and Information Technology (pp. 7-19). London: Taylor \& Francis.

Xiao, Y., Lasome, C., Moss, J., Mackenzie, C. F., \& Faraj, S. (2001). Cognitive properties of a whiteboard: A case study in a trauma centre. In W. Prinz, M. Jarke, Y. Rogers, K. Schmidt \& V. Wulf (Eds.), ECSCW '01: Proceedings of the European Conference on Computer Supported Cooperative Work (pp. 259-278). Dordrecht: Kluwer. 